\title{
Three novel mutations in Chinese patients with CSF1R-related leukoencephalopathy
}

\author{
Min Chu ${ }^{1 \#}$, Dong-Xin Wang ${ }^{1,2 \#}$, Yue Cui ${ }^{1}$, Yu Kong ${ }^{1}$, Li Liu ${ }^{1}$, Ke-Xin Xie ${ }^{1}$, Tian-Xinyu Xia ${ }^{1}$, Jing Zhang ${ }^{1}$, \\ Ran Gao ${ }^{1}$, Ai-Hong Zhou ${ }^{1}$, Chao-Dong Wang ${ }^{1}$, Li-Yong $\mathrm{Wu}^{1}$ \\ ${ }^{1}$ Department of Neurology, Xuanwu Hospital, Capital Medical University, Beijing, China; ${ }^{2}$ Department of Neurology, Shijiazhuang People's \\ Hospital, Shijiazhuang, China \\ Contributions: (I) Conception and design: LY Wu; (II) Administrative support: LY Wu; (III) Provision of study materials or patients: J Zhang, R Gao, \\ AH Zhou, CD Wang, LY Wu; (IV) Collection and assembly of data: M Chu, DX Wang, Y Cui, Y Kong, L Liu, KX Xie, TX Xia; (V) Data analysis \\ and interpretation: M Chu, DX Wang, Y Cui; (VI) Manuscript writing: All authors; (VII) Final approval of manuscript: All authors. \\ \#These authors contributed equally to this work. \\ Correspondence to: Li-Yong Wu, MD, PhD. Department of Neurology, Xuanwu Hospital, Capital Medical University, Beijing 100053, China. \\ Email: wmywly@hotmail.com.
}

Background: $C S F 1 R$-related encephalopathy refers to adult-onset leukodystrophy with neuroaxonal spheroids and pigmented glia (ALSP) due to CSF1R mutations, which is a rare autosomal dominant white matter disease including two pathological entities, hereditary diffuse leukoencephalopathy with spheroids (HDLS) and pigmentary orthochromatic leukodystrophy (POLD). The aim of this study was to identify additional causative mutations in the CSF1R gene and clarify their pathogenic effects.

Methods: Whole-exome sequencing was conducted for nine Chinese patients diagnosed with possible ALSP based on clinical and neuroimaging findings from March 2014 to June 2020 at Xuanwu Hospital (Beijing, China). Variant pathogenicity was assessed according to the American College of Medical Genetics and Genomics and Association for Molecular Pathology (ACMG/AMP) Standards and Guidelines.

Results: Mean \pm standard deviation (range) age of disease onset in the nine patients was 39.22 \pm 9.63 [25-54] years. Four of the nine patients were male, and four out of nine had a remarkable family history. Seven CSF1R mutations were identified in the nine patients; four (p.G17C, p.R579Q, p.I794T and c.2909_2910insATCA) have been previously reported, while three (p.V613L, p.W821R and c. $2442+2 \_2442+3$ dup T) were novel. Of the latter, two (p.V613L and p.W821R) were likely pathogenic and 1 (c.2442+2_2442+3dupT) was of uncertain significance according to ACMG/AMP criteria.

Conclusions: These findings expand the mutational spectrum of ALSP and provide a basis for future investigations on etiologic factors and potential management strategies for this disease.

Keywords: Adult-onset leukoencephalopathy with axonal spheroids and pigmented glia (ALSP); pigmented orthochromatic leukodystrophy (POLD); hereditary diffuse leukoencephalopathy with spheroid (HDLS); colony-stimulating factor 1 receptor $(C S F 1 R)$; novel mutation; mutational hotspot

Submitted Jan 15, 2021. Accepted for publication Apr 08, 2021.

doi: $10.21037 / \mathrm{atm}-21-217$

View this article at: http://dx.doi.org/10.21037/atm-21-217

\section{Introduction}

CSF1R-related leukoencephalopathy is adult-onset leukoencephalopathy with axonal spheroids and pigmented glia (ALSP) in patients positive for CSF1R gene mutation. ALSP is an autosomal dominant white matter disease characterized by progressive dementia, personality changes, parkinsonism, or seizures as primary clinical symptoms, and including hereditary diffuse leukoencephalopathy with axonal spheroids (HDLS) and pigmented orthochromatic leukodystrophy (POLD), which have many clinical and 
pathological similarities (1-3). To date, 147 patients with $C S F 1 R$-related leukoencephalopathy have been reported worldwide (4).

The colony-stimulating factor 1 receptor $(C S F 1 R)$ gene on chromosome $5 \mathrm{q} 32$, which was identified as a causative gene in HDLS in 2011, encodes a transmembrane tyrosine kinase receptor that is mainly expressed in microglia in the brain and participates in their development and maintenance (3). Mutations in $C S F 1 R$ may lead to microglia dysfunction and pathologic manifestations (5). Most CSF1R mutations have been reported in Europe, the United States, and Japan and include 94 missense mutations, 13 splicing mutations, 6 deletion/insertion mutations, 1 code shift mutation, and 1 nonsense mutation (4). Exons 12-22 of the CSF1R gene encode the tyrosine kinase domain (TKD) of $C S F 1 R$ and are hotspots for mutations leading to functional impairment of the receptor $(3,6)$. To our knowledge, at least 21 mutations have been reported in China, 18 located on exon 12-22 including 14 missense mutations, 1 nonsense mutation and 3 insertion/deletion mutations; A further three were intronic splicing mutations, located on intron 2 to 3 , intron 13 to 14 and intron 17 to 18 (4,7-14). Although novel variants are occasionally reported, neither the functional consequences of all known CSF1R mutations nor the full genetic spectrum of mutations causing ALSP have yet been fully elucidated.

In this study, we analyzed the genetic profile of nine Chinese patients diagnosed with possible ALSP in order to identify possible novel causative mutations in the CSF1R gene and evaluate their pathogenic effects. This article is presented in accordance with the Materials Design Analysis Reporting (MDAR) reporting checklist (available at http:// dx.doi.org/10.21037/atm-21-217).

\section{Methods}

\section{Patients}

The study was conducted in accordance with the Declaration of Helsinki (as revised in 2013). Approval of the study protocol and for ethical considerations was granted by the Ethics Committee and local Institutional Review Board of Xuanwu Hospital, Capital Medical University (approval number 2014019). Written informed consent was obtained from all participants or their guardians prior to the start of the study. All protocols were carried out in accordance with relevant guidelines and regulations for the use of human subjects in research.
Index patients were recruited as a continuous series of nine cases clinically diagnosed with possible ALSP at Xuanwu Hospital from March 2014 to June 2020, according to established criteria (15). A standardized clinical interview was conducted, family history recorded, and a physical examination performed at the time of admission, with 1-6 years of outpatient or telephone follow-up. All patients underwent routine laboratory testing and no abnormalities were detected. Four patients completed neurophysiologic examinations including Mini Mental State Examination and/or Montreal Cognitive Assessment. All patients underwent a magnetic resonance image (MRI) scan, and five underwent a computed tomography (CT) scan.

\section{Whole-exome sequencing}

Whole-exome sequencing was conducted using total DNA samples obtained from each patient using the SureSelect Human All Exon V6 Kit (Agilent Technologies, Santa Clara, $\mathrm{CA}$ ), according to the manufacturer's protocol. After library preparation and capture, pooled libraries were sequenced (Hiseq-2000; Illumina, San Diego, CA, USA). Sequence reads were aligned to the human genome (GRCh37/ hg19) and assembled for single nucleotide polymorphism calling and subsequent analysis using Burrows-Wheeler Aligner software (http://bio-bwa.sourceforge.net/). Variants were annotated using Realigner Target Creator in the Genome Analysis Toolkit (16) and ANNOVAR (17). EasyExonPrimer (18) was used to generate primers for PCR validation of exome sequencing derived CSF1R gene variants. Sanger sequencing of protein-coding regions was conducted according to standard protocols. PCR products were purified and then sequenced in both directions using the Big Dye Terminator v3.1 Cycle Sequencing Kit (Applied Biosystems, Foster City, CA, USA), and the resulting products purified and analyzed using an ABI3730xl Genetic Analyzer (Applied Biosystems). DNA sequence variants were identified using Seq-Pilot software (JSI, Kippenheim, Germany) and named based on known sequences (accession numbers NM_005211.3 and NP_005202.2).

\section{Pathogenicity prediction analyses of CSF1R variants}

In silico prediction of the functional effects of missense mutations was carried out using Polymorphism Phenotyping v2 (PolyPhen2) (19), Sorting Intolerant From Tolerant (SIFT) (20), Mutationtaster (21) and the likelihood ratio test (LRT) (22). Protein sequence alignment was performed 


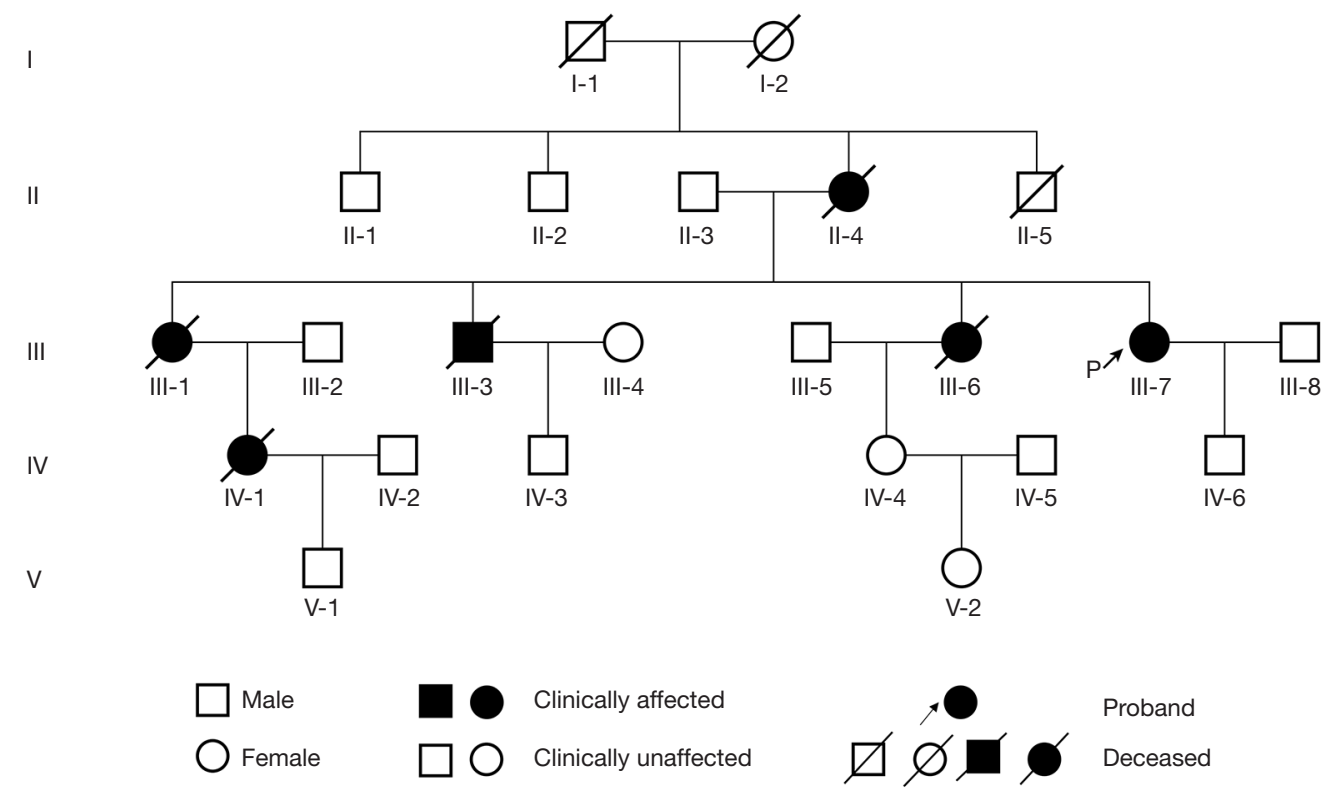

Figure 1 Pedigree of Family 1 with ALSP. The family pedigree included 23 individuals across five generations. Females and males are represented by circles and squares, respectively. Black and white symbols represent clinically affected and unaffected individuals, respectively. Diagonal lines indicate a deceased individual. The proband is indicated by an arrowhead. The proband's mother (II-4), siblings (III-1, III-3, and III-6), and niece (IV-1) were clinically affected but all passed away.

with UniProt (https://www.uniprot.org) to determine whether sequences were evolutionarily conserved across different species including Mus musculus (mouse), Rattus norvegicus (rat), Danio rerio (zebrafish), Canis lupus familiaris (dog), Bos taurus (bovine), and Macaca mulatta (rhesus macaque). Variant frequency was evaluated using Genome Aggregation Database (gnomAD), 1000 Genomes Project, and Exome Aggregation Consortium databases. All novel variants were classified as pathogenic/likely pathogenic, benign/likely benign, or uncertain significance, according to the 2015 American College of Medical Genetics and Genomics and Association for Molecular Pathology (ACMG/AMP) Standards and Guidelines (23). All analyses were performed on the Seqmax (https://www.seqmax.com/; accessed March 2020) and Pubvar (https://www.pubvar. com; accessed March 2020) platforms.

\section{Statistical analyses}

Continuous data were represented as means \pm standard deviation, dichotomous data were as values. Mean age of onset between male and female were compared via $t$-test. All statistical analyses were performed using IBM SPSS
Statistics $^{\circledR}$ v22.0.0.0 (SPSS Inc., Chicago, IL, 2013). P values less than 0.05 were regarded as statistically significant.

\section{Results}

\section{Demographic and clinical characteristics of patients}

A series of nine ALSP patients with CSF1R mutations from independent families were included in our study. Four of the nine $(44.4 \%)$ patients were male. Mean \pm standard deviation (range) age of disease onset in the nine patients was $39.22 \pm 9.63[25-54]$ years. No significant difference was found in the mean age at onset between male and female patients (male vs. female, $33.50 \pm 8.06$ vs. $43.80 \pm 8.82$ years; $\mathrm{P}=0.114$ ). Four patients had a remarkable family history that suggested an autosomal dominant pattern of inheritance. Pedigree analysis of the family of Patient 1 including 23 individuals from five generations was conducted (Figure 1). The patient's mother (II-4), siblings (III-1, III-3, and III-6), and niece (IV-1) were clinically affected but had all died, while her 32-year-old son (IV-6) did not show any symptoms. The mother of Patient 2 had had symptoms similar to the proband but was also deceased. The family history of two other patients (Patients 8 and 9) have been previously reported (11) and are 
not described in detail here.

The clinical characteristics of the patients are summarized in Table 1. Six of the nine patients presented with cognitive decline, one had personality change, one had parkinsonism, and one had walking difficulties as initial symptoms. Seven of the nine patients presented with cognitive decline, seven with pyramidal signs, and four with parkinsonism; two had personality changes, and one had epilepsy as clinical symptoms.

Brain MRI scans revealed that all patients had white matter lesions and brain atrophy; six had thinning of the corpus callosum, three had enlarged ventricles, and two had persistent limited diffusion on diffusion-weighted imaging (DWI). The brain MRI scans of Patients 1, 2, and 4 are shown in Figure 2. Four of the five patients who underwent CT scanning and had subcortical calcifications (see Figures $\mathrm{S} 1, \mathrm{~S} 2)$.

All patients were followed up for 1-6 years; six are still alive and three had died at the last follow-up. For the three deceased patients, mean \pm standard deviation disease duration from onset until death was $4.33 \pm 2.01$ years $(2,5$, and 6 years), and all experienced progressive deterioration of brain function and became mute and bedridden, with incontinence and dysphagia, and required nasogastric intubation at the terminal stage of illness.

\section{Genetic analysis}

Seven CSF1R mutations were detected in our nine ALSP patients, including three novel variants (p.V613L, p.W821R and c. $2442+2 \_2442+3$ dupT) and four known mutations (p.G17C, p.R579Q, p.I794T and c.2909_2910insATCA). Notably, p.I794T was present in three patients (Patients 7, 8 , and 9). Genetic screening results and protein sequence alignments with other vertebrate species are shown in Figure 3.

The three novel variants and prediction of their pathogenicity are summarized in Table 2. Two missense mutations (p.V613L and p.W821R) affected the functional TKD of CSF1R (PM1) and were absent in healthy population (PM2). These mutations are in residues highly conserved across species and computational predictions support a deleterious effect (PP3). As the phenotypes and family histories of the patients indicated a disease with single-gene etiology (PP4), the variants were interpreted as likely pathogenic $(\mathrm{PM} 1+\mathrm{PM} 2+\mathrm{PP} 3+\mathrm{PP} 4)$. The intronic splice-site mutation c. $2442+2 \_2442+3$ dupT was determined to be of uncertain significance (PM2). Thus, according to ACMG/AMP guidelines for sequence variant interpretation, the p.V613L and $\mathrm{p} . \mathrm{W} 821 \mathrm{R}$ mutations were predicted to be likely pathogenic, whereas c. $2442+2 \_2442+3$ dupT was interpreted as of uncertain significance.

The three known mutations are not described in detail here. The p.I794T mutation (accession no. 29813) is recorded in clinVar (https://www.ncbi.nlm.nih.gov/clinvar/) as a pathogenic variant, while p.G17C, p.R579Q and c.2909_2910insATCA are likely pathogenic, as previously reported $(11,13)$.

\section{Discussion}

In this study, we identified three novel mutations in the CSF1R gene in Chinese patients with ALSP, including two missense mutations [p.V613L (exon 13) and p.W821R (exon 19) and one splicing site mutation (c.2442+2_2442+3dupT (intron 17-18)]. Among them, two were likely pathogenic variants [p.V613L (exon 13) and p.W821R (exon 19)] and one was a variant of uncertain significance [c.2442+2_2442+3dupT (intron 17-18)].

The mean disease duration of our patients was 4.3 years, which was shorter than the average disease duration ( 6.8 years) previously reported (24). There are some possible explanations for this discrepancy. First, the mean age of onset was 39.2 years in our patients, compared with 43 years in previously reported patients, and it is established that neurodegenerative genetic diseases may progress rapidly if onset occurs at an early age. Second, mean disease duration was calculated from a very small number of patients (only three) who died during our study. Finally, the previously reported sample comprised 26 Japanese patients with ALSP and 96 symptomatic carriers, based on reports published worldwide, whereas we focused on only Chinese patients in our institution; hence it is possible that regional, medical care condition, and/or ethnic variations contributed to the observed difference. Sex-dependent structural and functional differences in microglia have been convincingly demonstrated in some experimental studies, and female patients with CSF1R-related leukoencephalopathy were reported to develop clinical symptoms significantly earlier than males in some studies (24-26); however, no significant difference was found according to sex in our study, possibly because of the small sample size.

The two identified missense mutations [p.V613L (exon 13) and p.W821R (exon 19)] are located in the CSF1R gene mutation hotspot at exons 12-22, which encodes the TKD. Thus, these amino acid substitutions could perturb 


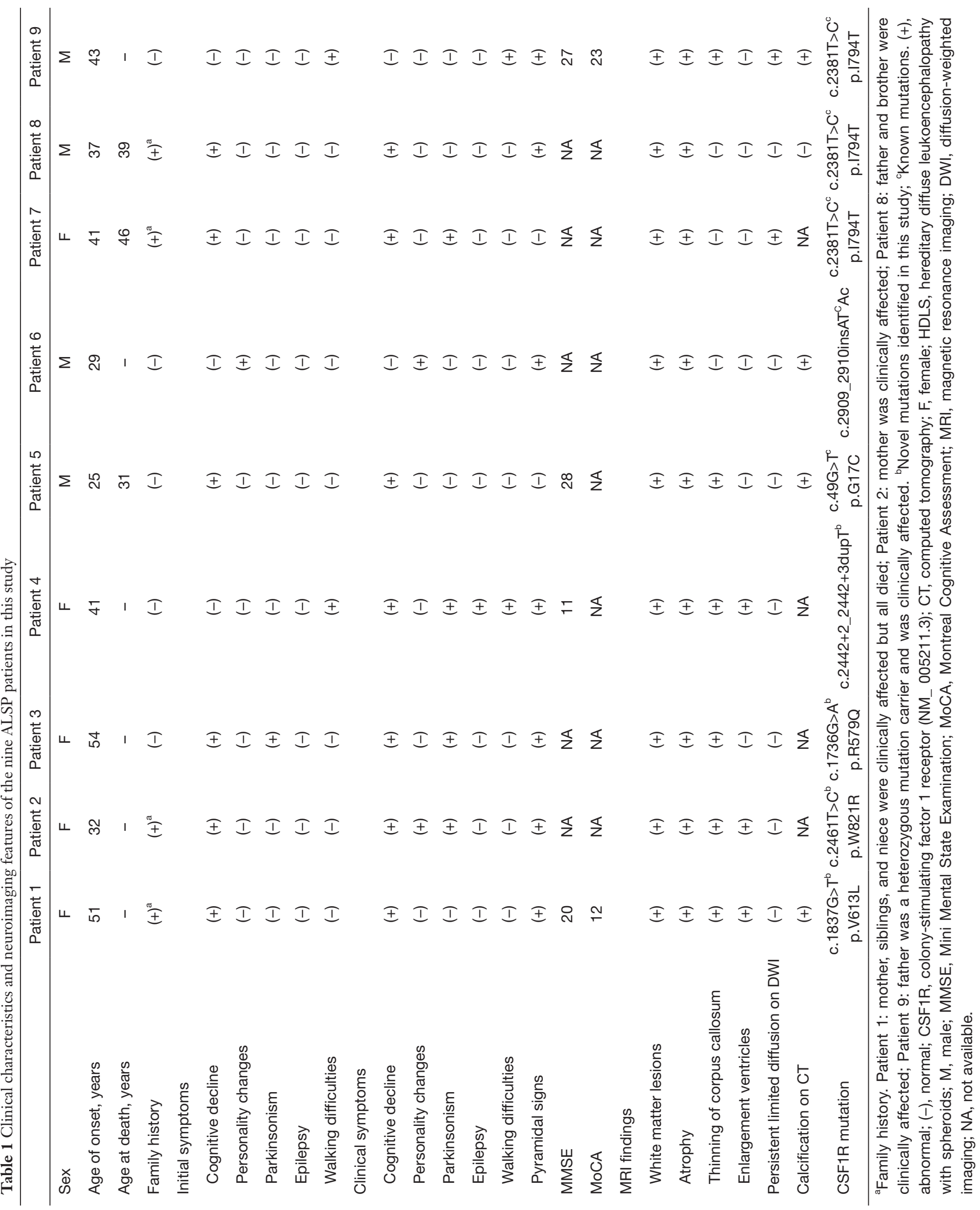




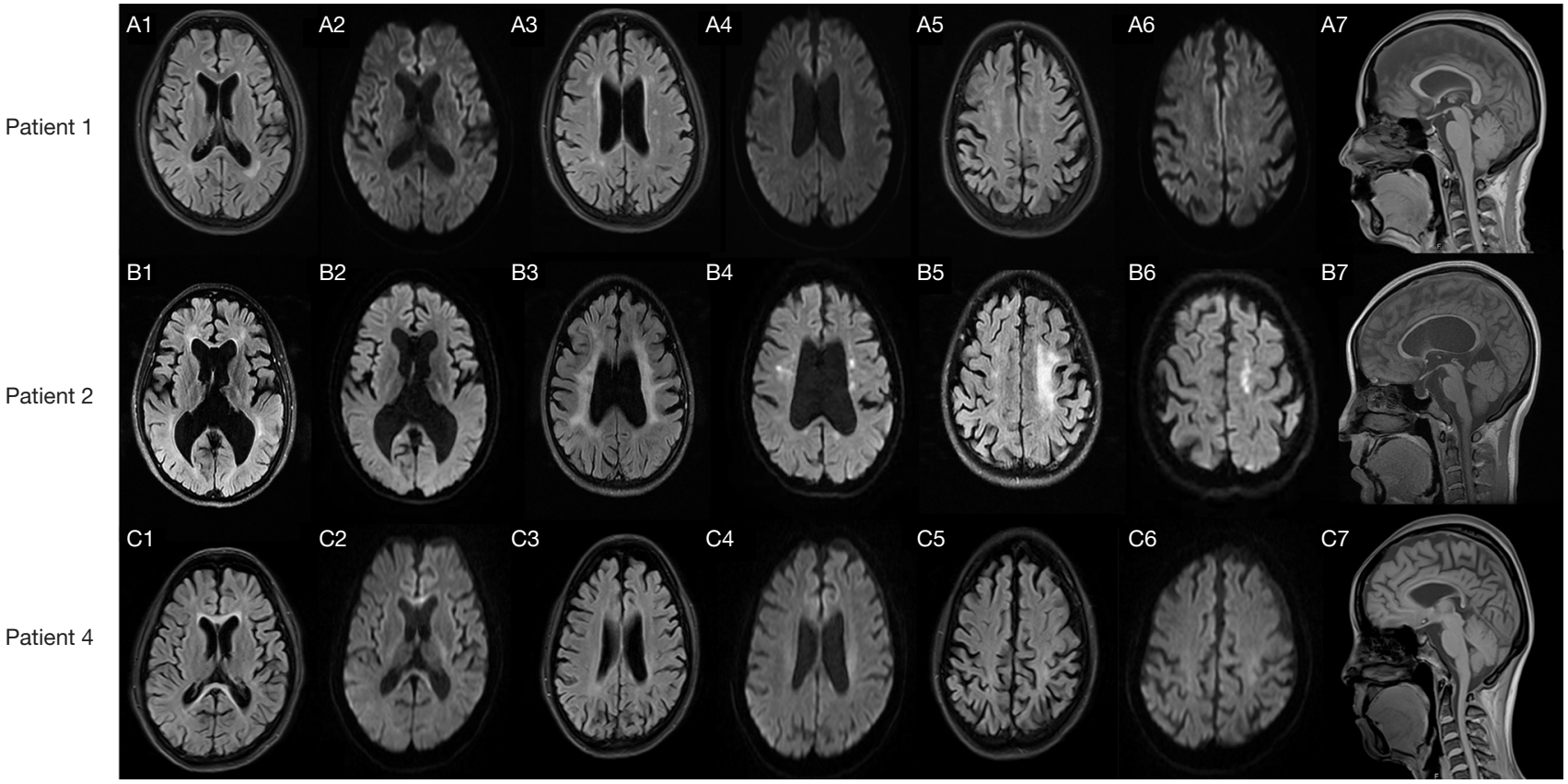

Figure 2 Brain MRI findings of three patients with ALSP. The first and second columns show the T2 fluid-attenuated inversion recovery (FLAIR) and corresponding diffusion-weighted imaging (DWI) images at the level of the third and lateral ventricles. The third and fourth columns show T2 FLAIR and DWI images at the level of the lateral ventricles. The fifth and sixth columns show T2 FLAIR and DWI images at the level of the centrum semiovale. The last column show T1-weighted images in the sagittal view. Images A1-A7 (Patient 1, 2 years after disease onset) revealed bilateral periventricular leukodystrophy and global brain atrophy, especially in the frontal and temporal lobes. Images B1-B7 (Patient 2, 7 months after disease onset) show asymmetric confluent T2 hyperintensities in periventricular regions and centrum semiovale, with some areas of restricted diffusion; apparent generalized cerebral atrophy of central and cortical gray matter and enlargement of the lateral ventricles; and thinning of the corpus callosum. Images C1-C7 (Patient 4, 6 months after disease onset) show prominent hyperintensities in the corpus callosum and brain atrophy.

the structure of the CSF1R protein, resulting in disease (PM1). Further, these mutations were absent from the gnomAD, 1000 Genomes Project and Exome Aggregation Consortium databases (PM2). Additionally, amino acids in this region are highly conserved across species, and the identified variants were predicted to have disease-damaging effects (probably damaging, damaging, disease-causing and deleterious) using four in silico analysis tools (PolyPhen-2, SIFT, Mutationtaster, and LRT) (PP3). Patients 1 and 2, harboring p.V613L and p.W821R, respectively, had the classic manifestations of ALSP, including memory deficit, speech dysfunction, and white matter lesions, according to neuroimaging, as well as remarkable family history (PP4); hence, the variants identified thus met the criteria for likely pathogenic (two pieces of moderate and two pieces of supporting evidence).

The splice-site variant, c. $2442+2 \_2442+3$ dupT, was absent from healthy populations, according to searches of
gnomAD, 1000 Genomes Project, and Exome Aggregation Consortium databases (PM2); however, it did not meet any other ACMG/AMP criteria for pathogenicity and was therefore considered as a variant of uncertain significance (one piece of moderate evidence). Nonetheless, as reported previously, mutations on intron 17 to 18 have frequently been associated with functional impacts, harboring half $(5 / 10)$ of the known splice-site variants associated with ALSP (5,27-31). We also identified c.2442+2_2442+3dupT, a +2 splice-site mutation comprising a duplicated thymidine adjacent to the guanine thymidine splice donor of intron 17 to18; whether or not this mutation causes a deleterious effect on the wide-type donor site splicing signal remains unknown. Previous functional studies provide some evidence supporting its potential influence on splicing of the $C S F 1 R$ gene transcript or causing exon 18 skipping and functional impairment of CSF1R, as previously reported for c. $2442+2 \mathrm{~T}>\mathrm{C}(29,30)$; Moreover, CSF1R expression levels 
A

Patient 1 p.V613L (c.1837G >T)

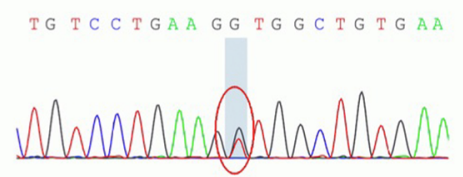

Homo sapiens DAVLK V AVKMLKSTAHADEKEA Mus musculus DAVLK V AVKMLKSTAHADEKEA Rattus norvegicus DAVLK V AVKMLKSTAHADEKEA Danio rerio DN ITR V AVKMLKASAHPDEREA Canis lupus DAVLK V AVKMLKSTAHADEKEA Bos taurus DAVLK V AVKMLKSTAHADEKEA

B Macaca mulatta DAVLK V AVKMLKSTAHADEKEA
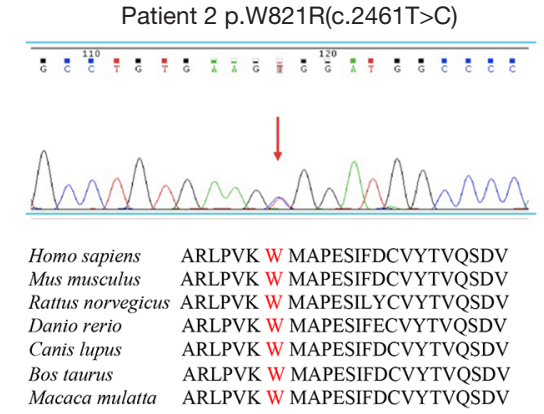

C

Patient 3 p.R579Q (c.1736G>A)

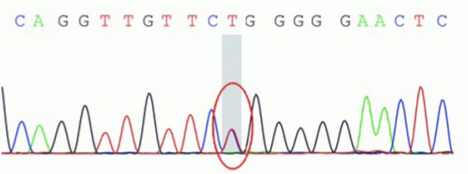

Homo sapiens EKWEFP R NNLQFGKTLGAGAFGKV Mus musculus EKWEFP R NNLQFGKTLGAGAFGKV Rattus norvegicus EKWEFP R NNLQFGKTLGAGAFGKV Danio rerio EKWEFP R DKLKLGKTLGAGAFGKV Canis lupus EKWEFP R NNLKLGKTLGAGAFGKV Bos taurus EKWEFP R NNLQFGKTLGAGAFGKV Macaca mulatta EKWEFP R NNLQFGKTLGAGAFGKV

D Patient 4 c.2442+2_2442+3dupT
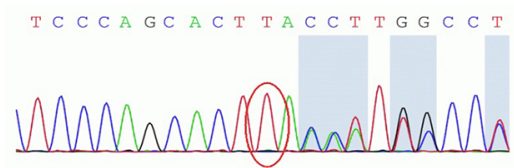

ACCTGGACAAGGAGGATGGACGGCCCCT GGAGCTCCGGGACCTGCTTCACTTCTCC AGCCAAGTAGCCCAGGGCATGGCCTTCC TCGCTTCCAAGAAT

intron 17-18 gtgagtaggaacct..........cctgcagtgctttccctcag

intron 17-18 gttgagtaggaacct..........cctgcagtgctttccctcag
E

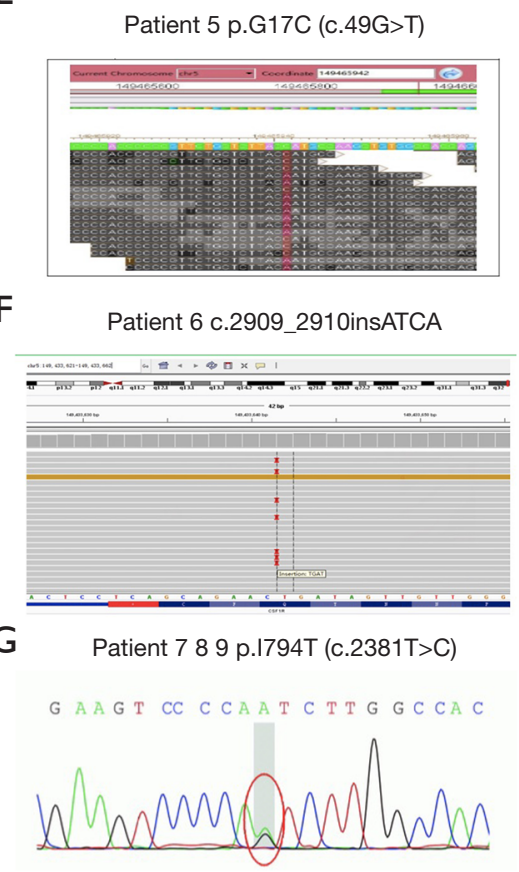

Figure 3 CSF1R gene mutation analysis. (A,B,C) Sequencing chromatograms showing three novel missense mutations of CSF1R (p.V613L, p.R579Q, and p.W821R). Protein sequence alignment was performed for parts of the TKD to evaluate conservation across species including Mus musculus (mouse), Rattus norvegicus (rat), Danio rerio (zebrafish), Canis lupus familiaris (dog), Bos taurus (bovine), and Macaca mulatta (rhesus macaque). (D) Sequencing chromatogram showing a novel splice site mutation of CSF1R (c.2442+2_2442+3dupT). At the beginning of intron 17 to 18 , a duplicated thymidine potentially alters the donor site splice signal, likely affecting splicing of the CSF1R gene transcript. (E,F,G) Results of sequence analysis showing three known heterozygous mutations of CSF1R (p.G17C, c.2909_2910insATCA, and p.I794T). NM_005211.3 and NP_005202.2 were used as reference cDNA and protein sequences, respectively.

were markedly reduced in the brain of patient with the c. $2442+1 \mathrm{G}>\mathrm{T}$ mutation (28), which is located in the same intron as c. $2442+2 \_2442+3$ dupT. Therefore, we speculate that c. $2442+2 \_2442+3$ dup T likely influence splicing, thereby causing pathogenic changes; however, as it is not a canonical splice site mutation, additional functional analyses will be required to address this hypothesis in the future.

Our study had some limitations. Firstly, the sample size was small because ALSP is a rare disease in China. Secondly, familial cosegregation (linkage) analyses were not carried out because all affected family members, other than the probands, were deceased and the surviving relatives refused genetic testing. Finally, in vitro functional verification of the intronic mutation was not performed; however, this analysis will be conducted in a future study.

In conclusion, we identified three novel mutations in the $C S F 1 R$ gene, two of which are likely pathogenic, and one uncertain significance. Our findings expand the mutational profile of ALSP and provide a basis for future research on the etiology and clinical management of this disease. 
Table 2 Pathogenicity prediction analyses of the three novel CSF1R variants identified in this study

\begin{tabular}{|c|c|c|c|}
\hline & Patient 1 & Patient 2 & Patient 4 \\
\hline Position & 149441075 & 149435682 & 150056217 \\
\hline CSF1R exon/intron & Exon 13 & Exon 19 & Intron 17-18 \\
\hline \multicolumn{4}{|l|}{ Variant effect } \\
\hline Amino acid change & p.V613L & p.W821R & NA \\
\hline Domain & Tyrosine kinase domain & Tyrosine kinase domain & NA \\
\hline \multicolumn{4}{|l|}{ Population allele frequency } \\
\hline Genome Aggregation Database & NR & NR & NR \\
\hline \multicolumn{4}{|l|}{ Functional prediction } \\
\hline Polyphen2 & Probably damaging & Probably damaging & NA \\
\hline SIFT & Damaging & Damaging & NA \\
\hline Mutation Taster & Disease-causing & Disease-causing & NA \\
\hline LRT & Deleterious & Deleterious & NA \\
\hline \multicolumn{4}{|l|}{ General prediction } \\
\hline CADD Phred score & 31 & 28.1 & NA \\
\hline ACMG/AMP criteria & Likely pathogenic & Likely pathogenic & Uncertain significance \\
\hline
\end{tabular}

ACMG/AMP, American College of Medical Genetics and Genomics and Association for Molecular Pathology; CADD, combined annotationdependent depletion; CSF1R, colony-stimulating factor 1 receptor (NM_ 005211.3); LRT, likelihood ratio test; NA, not available; NR, not reported; Polyphen2, Polymorphism Phenotyping v2; SIFT, sorting intolerant from tolerant.

\section{Acknowledgments}

We thank the patients and their families for granting us the permission to publish this information.

Funding: This work was supported by grants from the Ministry of Science and Technology of China [No. 2019YFC0118600]; National Natural Science Foundation of China [No. 81971011]; and Beijing Municipal Science and Technology Committee [Nos. D171100008217005, 7202060].

\section{Footnote}

Reporting Checklist: The authors have completed the MDAR reporting checklist. Available at http://dx.doi.org/10.21037/ atm-21-217

Data Sharing Statement: Available at http://dx.doi. org/10.21037/atm-21-217

Peer Review File: Available at http://dx.doi.org/10.21037/ atm-21-217

Conflicts of Interest: All authors have completed the ICMJE uniform disclosure form (available at http://dx.doi. org/10.21037/atm-21-217). The authors have no conflicts of interest to declare.

Ethical Statement: The authors are accountable for all aspects of the work in ensuring that questions related to the accuracy or integrity of any part of the work are appropriately investigated and resolved. The study was conducted in accordance with the Declaration of Helsinki (as revised in 2013). The study was approved by the Ethics Committee and local Institutional Review Board of 
Xuanwu Hospital, Capital Medical University (approval number 2014019) and informed consent was taken from all individual participants.

Open Access Statement: This is an Open Access article distributed in accordance with the Creative Commons Attribution-NonCommercial-NoDerivs 4.0 International License (CC BY-NC-ND 4.0), which permits the noncommercial replication and distribution of the article with the strict proviso that no changes or edits are made and the original work is properly cited (including links to both the formal publication through the relevant DOI and the license). See: https://creativecommons.org/licenses/by-nc-nd/4.0/.

\section{References}

1. Axelsson R, Röyttä M, Sourander P, et al. Hereditary diffuse leucoencephalopathy with spheroids. Acta Psychiatr Scand Suppl 1984;314:1-65.

2. Adams SJ, Kirk A, Auer RN. Adult-onset leukoencephalopathy with axonal spheroids and pigmented glia (ALSP): Integrating the literature on hereditary diffuse leukoencephalopathy with spheroids (HDLS) and pigmentary orthochromatic leukodystrophy (POLD). J Clin Neurosci 2018;48:42-9.

3. Konno T, Kasanuki K, Ikeuchi T, et al. CSF1R-related leukoencephalopathy: A major player in primary microgliopathies. Neurology 2018;91:1092-104.

4. Chen J, Luo S, Li N, et al. A Novel Missense Mutation of the CSF1R Gene Causes Incurable CSF1R-Related Leukoencephalopathy: Case Report and Review of Literature. Int J Gen Med 2020;13:1613-20.

5. Rademakers R, Baker M, Nicholson AM, et al. Mutations in the colony stimulating factor 1 receptor (CSF1R) gene cause hereditary diffuse leukoencephalopathy with spheroids. Nat Genet 2011;44:200-5.

6. Konno T, Miura T, Harriott AM, et al. Partial loss of function of colony-stimulating factor 1 receptor in a patient with white matter abnormalities. Eur J Neurol 2018;25:875-81.

7. Zhuang LP, Liu CY, Li YX, et al. Clinical features and genetic characteristics of hereditary diffuse leukoencephalopathy with spheroids due to CSF1R mutation: a case report and literature review. Ann Transl Med 2020;8:11.

8. Shi T, Li J, Tan C, et al. Diagnosis of hereditary diffuse leukoencephalopathy with neuroaxonal spheroids based on next-generation sequencing in a family: Case report and literature review. Medicine (Baltimore) 2019;98:e15802.

9. Tian WT, Zhan FX, Liu Q, et al. Clinicopathologic characterization and abnormal autophagy of CSF1Rrelated leukoencephalopathy. Transl Neurodegener 2019;8:32.

10. Du Q, Chen H, Shi Z, et al. A novel mutation in the CSF1R gene causes hereditary diffuse leukoencephalopathy with axonal spheroids. Neurol Sci 2019;40:1287-90.

11. Wu L, Liu J, Sha L, et al. Sporadic Cases with Novel Mutations and Pedigree in Hereditary Leukoencephalopathy with Axonal Spheroids. J Alzheimers Dis 2017;56:893-8.

12. Yang X, Huang P, Tan Y, et al. A Novel Splicing Mutation in the CSF1R Gene in a Family With Hereditary Diffuse Leukoencephalopathy With Axonal Spheroids. Front Genet 2019;10:491.

13. Bewernick BH, Kayser S, Gippert SM, et al. Deep brain stimulation to the medial forebrain bundle for depressionlong-term outcomes and a novel data analysis strategy. Brain Stimul 2017;10:664-71.

14. Wang $M$, Zhang X. A novel CSF-1R mutation in a family with hereditary diffuse leukoencephalopathy with axonal spheroids misdiagnosed as hydrocephalus. Neurogenetics 2019;20:155-60.

15. Konno T, Yoshida K, Mizuta I, et al. Diagnostic criteria for adult-onset leukoencephalopathy with axonal spheroids and pigmented glia due to CSF1R mutation. Eur J Neurol 2018;25:142-7.

16. McKenna A, Hanna M, Banks E, et al. The Genome Analysis Toolkit: a MapReduce framework for analyzing next-generation DNA sequencing data. Genome Res 2010;20:1297-303.

17. Wang K, Li M, Hakonarson H. ANNOVAR: functional annotation of genetic variants from high-throughput sequencing data. Nucleic Acids Res 2010;38:e164.

18. Wu X, Munroe DJ. EasyExonPrimer: automated primer design for exon sequences. Appl Bioinformatics 2006;5:119-20.

19. Adzhubei IA, Schmidt S, Peshkin L, et al. A method and server for predicting damaging missense mutations. Nat Methods 2010;7:248-9.

20. Choi Y, editor. A fast computation of pairwise sequence alignment scores between a protein and a set of singlelocus variants of another protein. ACM Conference on Bioinformatics; 2012.

21. Schwarz JM, Cooper DN, Schuelke M, et al. MutationTaster2: mutation prediction for the deepsequencing age. Nat Methods 2014;11:361-2. 
22. Chun S, Fay JC. Identification of deleterious mutations within three human genomes. Genome Res 2009;19:1553-61.

23. Richards S, Aziz N, Bale S, et al. Standards and guidelines for the interpretation of sequence variants: a joint consensus recommendation of the American College of Medical Genetics and Genomics and the Association for Molecular Pathology. Genet Med 2015;17:405-24.

24. Konno T, Yoshida K, Mizuno T, et al. Clinical and genetic characterization of adult-onset leukoencephalopathy with axonal spheroids and pigmented glia associated with CSF1R mutation. Eur J Neurol 2017;24:37-45.

25. Guneykaya D, Ivanov A, Hernandez DP, et al. Transcriptional and Translational Differences of Microglia from Male and Female Brains. Cell Rep 2018;24:2773-2783.e6.

26. Han J, Zhu K, Zhou K, et al. Sex-Specific Effects of Microglia-Like Cell Engraftment during Experimental Autoimmune Encephalomyelitis. Int J Mol Sci 2020;21:6824.

27. Guerreiro R, Kara E, Le Ber I, et al. Genetic analysis

Cite this article as: Chu M, Wang DX, Cui Y, Kong Y, Liu L, Xie KX, Xia TX, Zhang J, Gao R, Zhou AH, Wang CD, Wu LY. Three novel mutations in Chinese patients with CSF1R-related leukoencephalopathy. Ann Transl Med 2021;9(13):1072. doi: 10.21037/atm-21-217 of inherited leukodystrophies: genotype-phenotype correlations in the CSF1R gene. JAMA Neurol 2013;70:875-82.

28. Konno T, Tada M, Tada M, et al. Haploinsufficiency of CSF-1R and clinicopathologic characterization in patients with HDLS. Neurology 2014;82:139-48.

29. Kawakami I, Iseki E, Kasanuki K, et al. A family with hereditary diffuse leukoencephalopathy with spheroids caused by a novel c. $2442+2 \mathrm{~T}>\mathrm{C}$ mutation in the CSF1R gene. J Neurol Sci 2016;367:349-55.

30. Lee D, Yun JY, Jeong JH, et al. Clinical evolution, neuroimaging, and volumetric analysis of a patient with a CSF1R mutation who presented with progressive nonfluent aphasia. Parkinsonism Relat Disord 2015;21:817-20.

31. Konno T, Broderick DF, Mezaki N, et al. Diagnostic Value of Brain Calcifications in Adult-Onset Leukoencephalopathy with Axonal Spheroids and Pigmented Glia. AJNR Am J Neuroradiol 2017;38:77-83. 


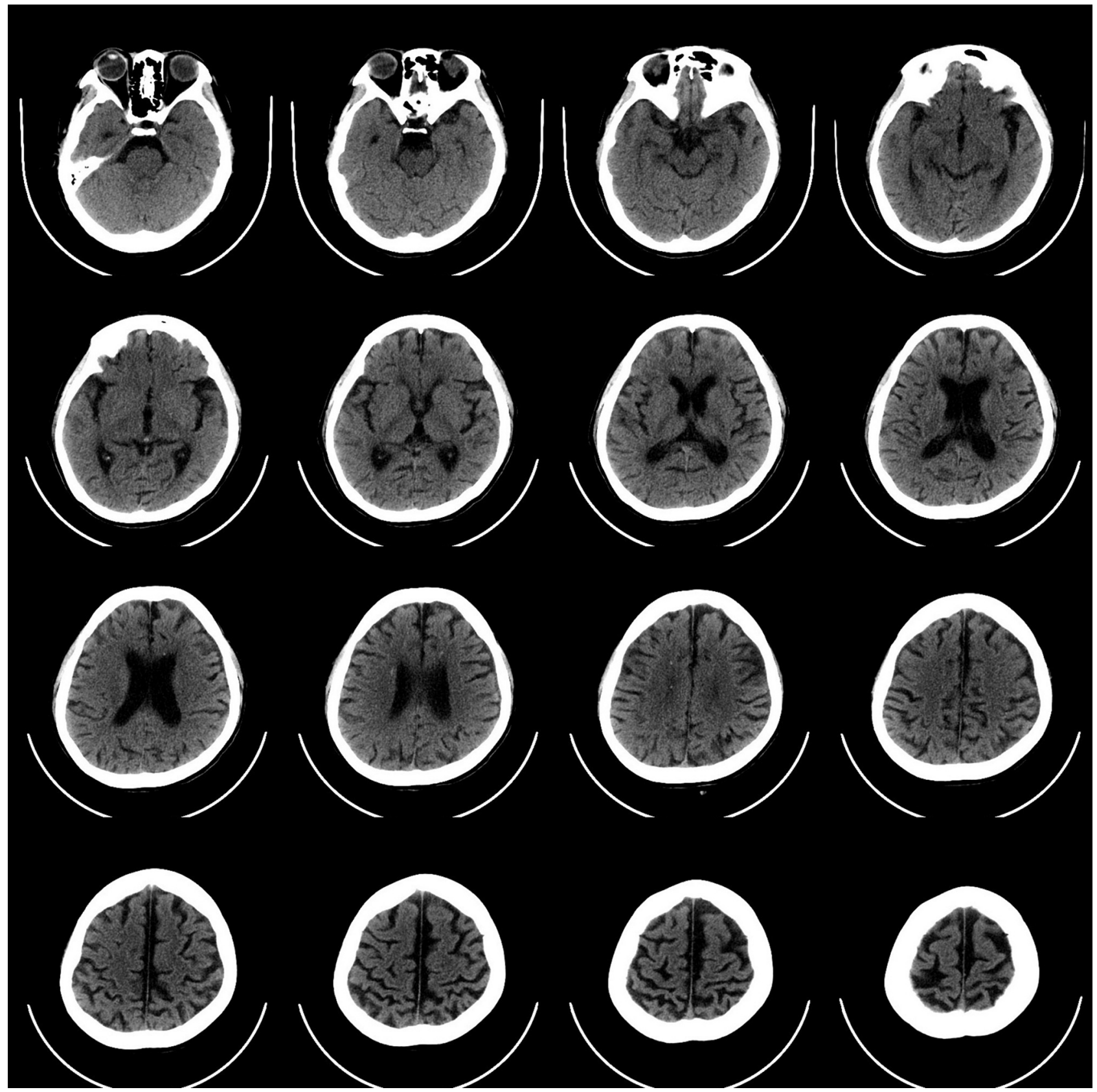

Figure S1 Brain CT images of patient 4. Calcifications are located at the frontal horns of the lateral ventricles, periventricular region and centrum semiovale. 


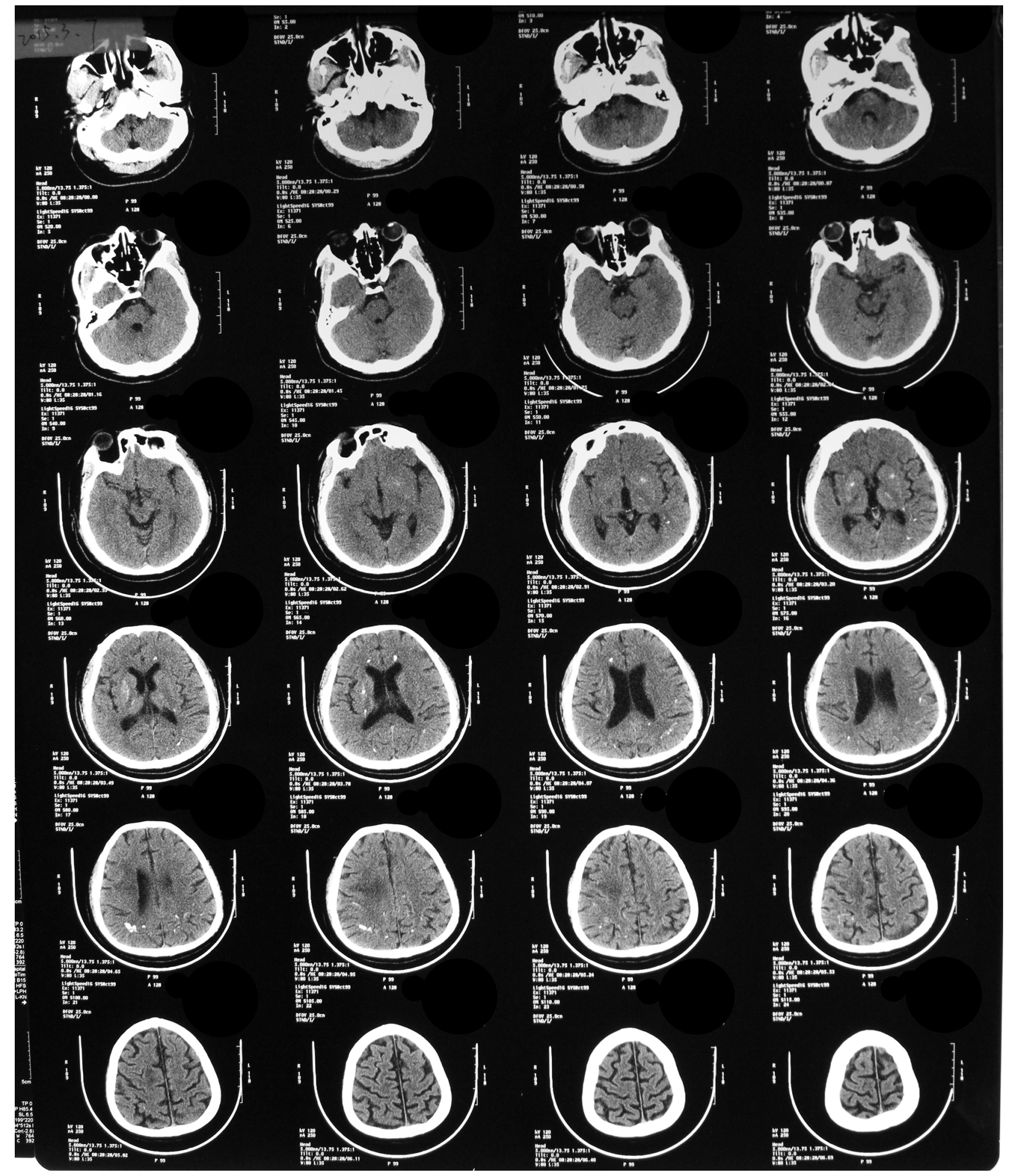

Figure S2 Brain CT images of patient 7. Predominant calcifications are located at extensive white matter regions including the frontal/ posterior horns of the lateral ventricles, periventricular and subcortical regions. 